\title{
Tratamento endovascular de ruptura traumática da aorta torácica descendente
}

\author{
Endovascular treatment of traumatic descending thoracic \\ aortic rupture
}

\begin{abstract}
João Roberto Breda, Ana Silvia Ragognetti Breda, Eliane Yumi Fujii, João Antonio Corrêa, Adriano Meneghini, Jaime Ignácio Jaramilo, Dirceu Rodrigues de Almeida, Adilson Casemiro Pires*
\end{abstract}

\begin{abstract}
Resumo
Paciente do sexo feminino, 55 anos de idade, vítima de atropelamento, foi admitida em unidade de emergência, onde se realizou o diagnóstico clínico, radiológico e tomográfico de ruptura traumática da aorta torácica descendente. Diante do achado, a paciente foi encaminhada para tratamento endovascular com colocação de endoprótese auto-expansível (stent) pela artéria femoral. O tratamento obteve sucesso, evidenciado pela exclusão da lesão localizada previamente no istmo aórtico. O tratamento endovascular tem sido indicado nas afecções de aorta torácica descendente com bons resultados iniciais. Na ruptura traumática de aorta, a terapêutica endovascular representa uma alternativa aceitável, especialmente devido aos riscos do tratamento operatório convencional.
\end{abstract}

Palavras-chave: Aorta lesões, aorta cirurgia, radiografia intervencionista, instrumentação.

\section{Introdução}

A utilização de endoprótese auto-expansível (stent) no tratamento das doenças da aorta torácica descendente tornou-se uma realidade nos últimos anos, sobretudo com o advento do acesso através da artéria femoral na sala de hemodinâmica ${ }^{1}$.

A busca e utilização desta alternativa terapêutica se justificam pela diminuição da morbidade e mortalidade cirúrgicas quando comparada com as técnicas tradicionais ${ }^{2}$. Deste modo, o tratamento endoluminal vem se tornando uma alternativa aceitável no tratamento das dissecções e aneurismas verdadeiros da aorta torácica

\begin{abstract}
A 55-year-old, female patient who was run over by a motor vehicle was admitted at an emergency room. Clinical, radiological and tomographic diagnosis of traumatic descending aortic thoracic rupture was performed. The patient was referred for endovascular treatment with placement of a self-expandable stent through the femoral artery. Treatment was successful, with exclusion of the lesion previously located in the aortic isthmus. Endovascular treatment has been indicated in the treatment of descending thoracic aortic diseases, with good initial results. In case of traumatic aortic rupture, endovascular treatment is a feasible alternative, especially due to risks offered by the conventional surgical treatment.
\end{abstract}

Keywords: Aortic injuries, aortic surgery, interventional radiography, instrumentation.

descendente. Nas rupturas traumáticas de aorta, o tratamento endovascular procura diminuir a morbidade e a mortalidade associadas ao tratamento operatório convencional.

O objetivo deste trabalho é relatar uma indicação de tratamento endovascular da ruptura traumática da aorta torácica descendente, localizada no istmo aórtico, em paciente vítima de atropelamento, com trauma de tórax e mecanismo de lesão por desaceleração.

\section{Relato de caso}

Mulher, 55 anos de idade, foi encaminhada ao serviço de emergência, vítima de atropelamento, com

* Disciplina de Cirurgia Cardiovascular, Faculdade de Medicina do ABC, Santo André, SP.

Trabalho realizado no Hospital Estadual Mário Covas, São Paulo.

Artigo submetido em 14.01.07, aceito em 09.04.07. 
diagnóstico de politraumatismo, tendo sido iniciadas as medidas de atendimento primário preconizadas e padronizadas. Após a estabilização do quadro e durante a avaliação secundária, diagnosticou-se o alargamento do mediastino. Mantido o quadro de estabilidade clínica, a paciente foi encaminhada para realização de tomografia computadorizada de tórax.

A tomografia de tórax confirmou o diagnóstico de ruptura traumática de aorta torácica descendente, com lesão localizada no istmo aórtico, além da presença de derrame pleural bilateral (Figura 1).

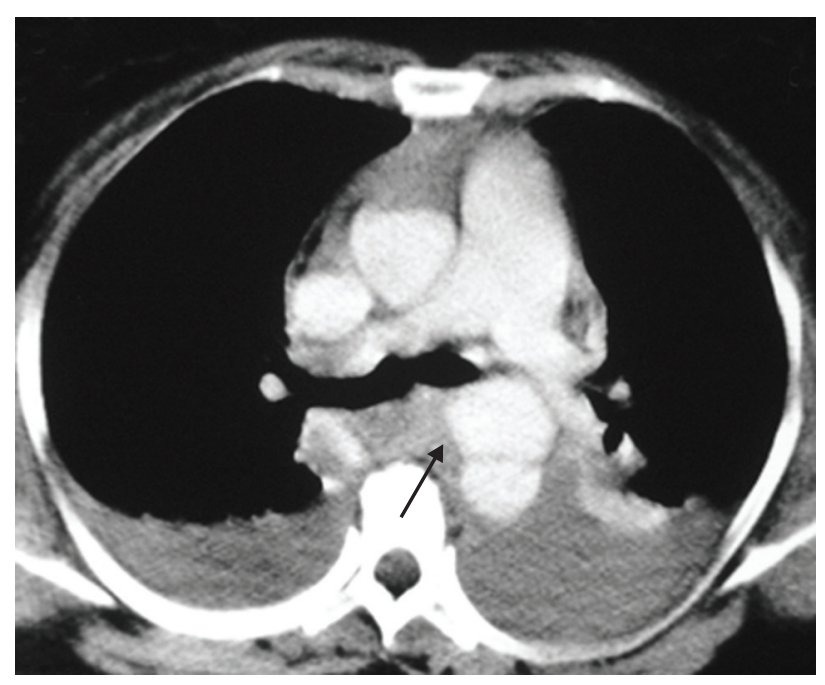

Figura 1 - Ruptura de aorta torácica descendente e derrame pleural bilateral

Com esse diagnóstico, a paciente foi encaminhada para o laboratório de hemodinâmica e submetida à aortografia. Diante do achado angiográfico e da gravidade da lesão, optamos pelo tratamento endovascular na própria sala de hemodinâmica, através da colocação de stent aórtico. O stent aórtico utilizado em nossa instituição é aquele atualmente fabricado pela indústria Braile-Biomédica, desenvolvido na Escola Paulista de Medicina (Universidade Federal de São Paulo).

O procedimento foi realizado com anestesia geral. A monitorização envolveu oximetria contínua, pressão arterial média e eletrocardiograma de superfície, sendo que o controle da inserção da prótese na luz aórtica foi realizado através da angiografia. Realizou-se o isola- mento cirúrgico da artéria femoral esquerda, escolhida para inserção da endoprótese e, após administração de 5.000 unidades de heparina por via endovenosa, foi feita arteriotomia femoral entre duas pinças vasculares.

Assim, o cateter contendo a endoprótese foi delicadamente introduzido no sistema ilíaco-femoral. A progressão do dispositivo foi controlada através da radioscopia. Uma vez determinada a posição correta da prótese no interior da aorta, o stent foi liberado através do recuo da bainha do cateter com controle da pressão arterial média (mantida entre 60 ou $70 \mathrm{mmHg}$ ), com o dispositivo colocado sob o óstio da artéria subclávia esquerda.

Após a liberação da endoprótese, foi realizada uma nova aortografia para avaliação do resultado, com exclusão completa do ponto de ruptura da aorta, denotando sucesso do procedimento. Não ocorreu oclusão completa da artéria subclávia (Figura 2). A drenagem pleural à direita foi realizada após o procedimento, devido ao grande derrame pleural com restrição pulmonar e dificuldade ventilatória associada ao quadro, com saída de líquido hemorrágico. A paciente foi então encaminhada à Unidade de Pós-Operatório, onde permaneceu monitorizada e com controle rigoroso das suas funções vitais.

No pós-operatório, antes da alta hospitalar, a paciente realizou a tomografia computadorizada de controle, evidenciando o sucesso da terapêutica, devido à ausência de extravasamento de contraste na região do istmo aórtico (Figura 3). Notou-se a presença de derrame pleural bilateral volumoso, apesar da drenagem pleural à direita. Discutiu-se a necessidade de toracotomia exploradora para limpeza e remoção de coágulos da cavidade. Porém, devido à gravidade do caso, optou-se por conduta expectante e, devido à boa evolução clínica, não foi indicado tratamento operatório adjuvante neste momento, sendo a paciente encaminhada para seguimento ambulatorial.

\section{Comentários}

O tratamento dos aneurismas de aorta com próteses intraluminais foi iniciado com o conceito introduzido por Dotter, em 1969. Em 1991, Parodi et al. divulgaram 


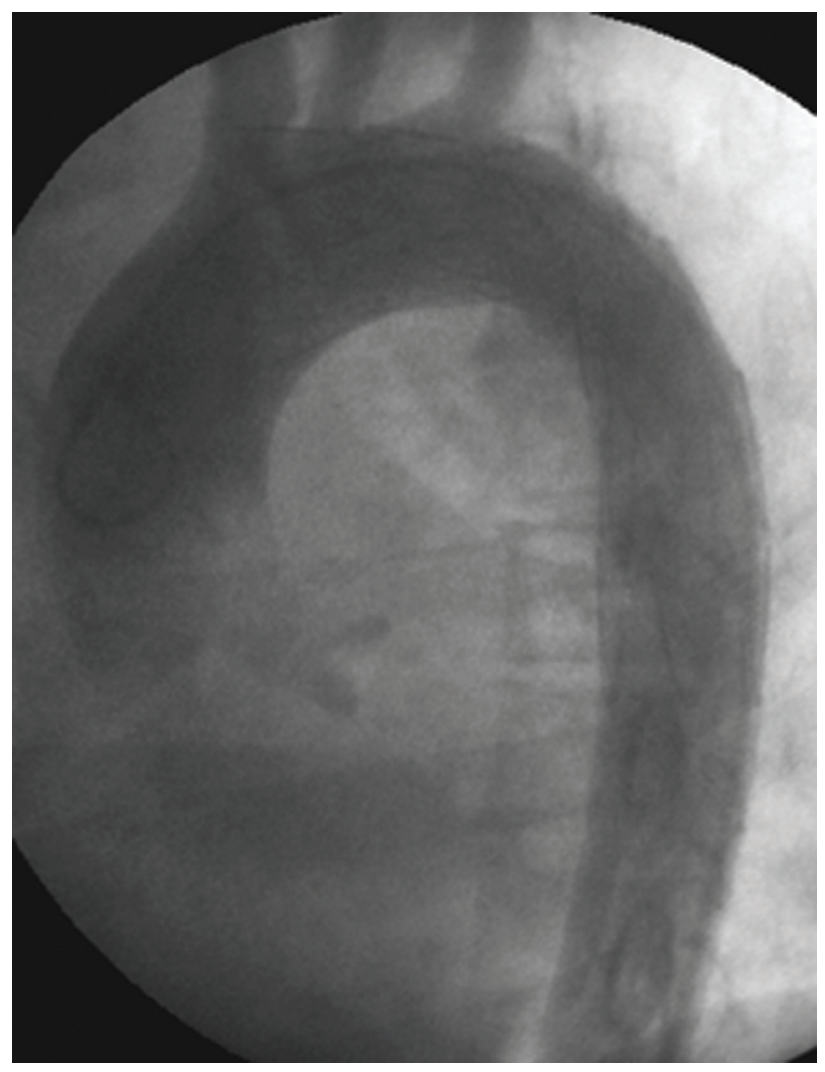

Figura 2 - Colocação do stent sob ruptura de aorta, sem a oclusão total da artéria subclávia esquerda e com a exclusão da lesão

amplamente o tratamento percutâneo, publicando o sucesso obtido com o uso de próteses expandidas por balão no tratamento dos aneurismas da aorta abdominal ${ }^{2}$. Em 1994, Dake et al. publicaram sua experiência na correção dos aneurismas da aorta torácica descendente usando próteses auto-expansíveis ${ }^{1}$.

As endopróteses de aorta representam uma opção para o tratamento dos aneurismas e dissecções da aorta torácica descendente. Por isso, vários centros ao redor do mundo têm estudado e publicado seus resultados com esta opção terapêutica ${ }^{2}$.

No Hospital de Ensino da Faculdade de Medicina do ABC, iniciamos nossa experiência em 1996, e desde então temos utilizado esta técnica no tratamento das dissecções e aneurismas da porção descendente da aorta torácica ${ }^{3}$. A ruptura traumática da aorta torácica descendente, relatada neste trabalho, não fazia parte dos diagnósticos que motivaram a indicação do tratamento endovascular na nossa instituição até a presente data.

$\mathrm{Na}$ literatura, existem relatos de indicações não usuais de terapêutica endovascular em afecções da porção torácica descendente da aorta. Shimono et al. relataram o tratamento endovascular de um único caso de dissecção aguda de aorta ascendente com fenda intimal na aorta descendente, onde, após a colocação da endoprótese, ocluindo o orifício intimal na porção descendente, obtiveram sucesso no tratamento, constatado através da trombose da falsa luz, tanto na aorta ascendente quanto na aorta descendente, com satisfatória evolução clínica do paciente ${ }^{4}$.

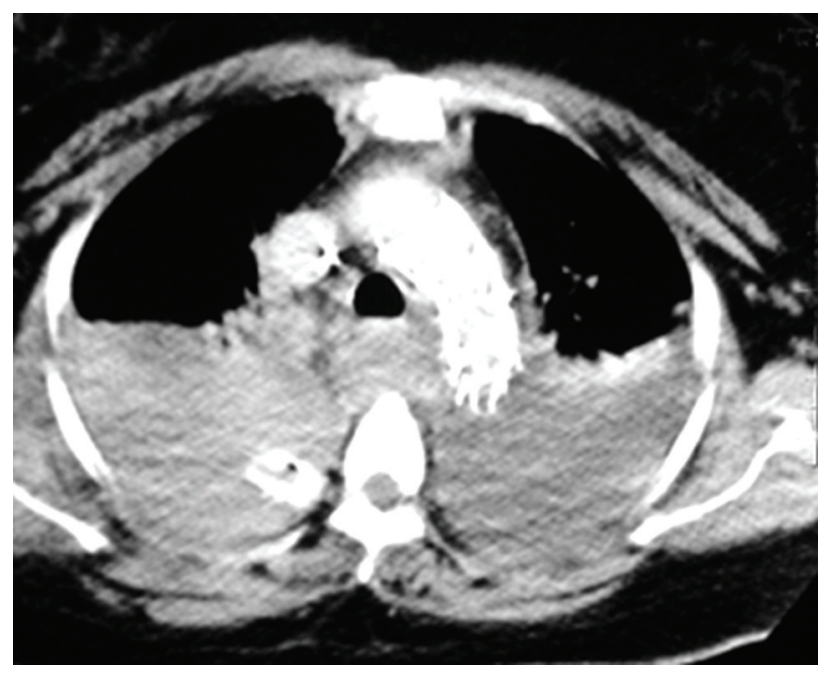

Figura 3 - Tomografia computadorizada de controle com a correção da lesão com stent

A ruptura da aorta descendente após trauma de tórax apresenta mortalidade imediata variando de $75 \%$ a $90 \%$ dos casos e, apesar dos avanços no tratamento operatório convencional, a mortalidade pode atingir $50 \%$ dos casos diagnosticados ${ }^{5}$. Deste modo, alternativas menos invasivas têm sido propostas para o tratamento desta grave situação clínica ${ }^{6,7}$. Em trabalho retrospectivo, Rosseau et al., analisaram 70 pacientes com ruptura traumática de aorta, comparando os resultados obtidos entre três modalidades terapêuticas (conservador, operação convencional e implante de endoprótese aórtica). Os autores demonstraram uma 
menor mortalidade e menor risco de paraplegia no grupo tratado de forma endovascular ${ }^{6}$. Do mesmo modo, Agostinelli et al. relatam casos de 15 pacientes submetidos a tratamento endovascular imediato após diagnóstico de ruptura traumática de aorta, concluindo que o procedimento representa uma opção segura nesta grave condição clínica ${ }^{7}$.

Deste modo, o tratamento endovascular pode representar uma opção nesta situação, com bons resultados iniciais. Entretanto, serão necessários trabalhos adicionais para comprovar o papel das endopróteses na prevenção de ruptura aórtica tardia, bem como o seguimento das possíveis complicações das endopróteses empregadas em indivíduos jovens e com diâmetro da aorta reduzido.

\section{Referências}

1. Dake MD, Miller DC, Semba CP, Mitchell RS, Walker PJ, Liddell RP. Transluminal placement of endovascular stent-grafts for the treatment of descending thoracic aortic aneurysms. N Engl J Med. 1994;331:1729-34.
2. Buffolo E, Palma H. Surgical treatment of type B dissecting aneurysms: what is new? Arch Chir Thorac Cardiovasc. 1997;19:171-2.

3. Breda JR, Almeida DR, Ramos Filho RA, Silas MG, Pires AC. Experience with utilization of auto-expandable stents introduced through the femoral artery for treatment of thoracic aortic diseases. Rev Bras Cir Cardiovasc. 2003; 18:129-32.

4. Shimono T, Kato N, Tokui T, et al. Endovascular stent-graft repair for acute type $\mathrm{A}$ aortic dissection with an intimal tear in the descending aorta. J Thorac Cardiovasc Surg. 1998;116:171-3.

5. Williams JS, Graff JA, Uku JM, Steinig JP. Aortic injury in vehicular trauma. Ann Thorac Surg. 1994;57:726-30.

6. Rousseau H, Dambrin C, Marcheix B, et al. Acute traumatic aortic rupture: a comparison of surgical and stent-graft repair. J Thorac Cardiovasc Surg. 2005;129:1050-5.

7. Agostinelli A, Saccani S, Borrello B, Nicolini F, Larini P, Gherli T. Immediate endovascular treatment of blunt aortic injury: our therapeutic strategy. J Thorac Cardiovasc Surg. 2006;131:1053-7.

Correspondência:

João Roberto Breda

Rua Antonio Bastos, 755/31

CEP 09040-220 - Santo André, SP

Tel.: (11) 4438.4311

E-mail: jbreda@hotmail.com 